\title{
Barreiras e Facilitadores do Processo Ensino-Aprendizagem de Estudantes de Medicina na Atenção Primária, no Município de São Paulo
}

\section{Barriers and Facilitators of the Teaching-Learning Process of Medical Students in Primary Care in the City of São Paulo}

\author{
Moniquelly Barbosa da Silva ${ }^{I}(\mathbb{D}$ \\ Izabel Rios ${ }^{I, I}(\mathbb{D})$ \\ Pedro Félix Vital Júnior ${ }^{I}$ (iD \\ Andréa Tenório Correia da Silva ${ }^{I, I}{ }_{(\mathbb{D})}$
}

\section{PALAVRAS-CHAVE}

- Educação Médica.

- Atenção Primária à Saúde.

- Aprendizagem.

- Pessoal de Saúde.

- Agentes Comunitários de Saúde.

- Pesquisa Qualitativa.
${ }^{\mathrm{I}}$ Faculdade Santa Marcelina, São Paulo, São Paulo, Brasil.

${ }^{\text {II }}$ Universidade de São Paulo, São Paulo, São Paulo, Brasil.

Introdução: Apesar de a atenção primária à saúde (APS) ser essencial para a formação médica, as percepçães dos profissionais das equipes de saúde da família (eSF) sobre o processo ensino-aprendizagem foram pouco estudadas, em particular em municípios nos quais o modelo de gestão da APS é indireto, como na cidade de São Paulo. O objetivo deste estudo foi analisar as percepções de profissionais das eSF sobre as barreiras e os facilitadores do processo ensino-aprendizagem dos estudantes de medicina na APS, no município de São Paulo. Método: Trata-se de uma pesquisa qualitativa. Foram realizadas entrevistas semiestruturada com 12 profissionais das eSF (quatro médicos, quatro enfermeiros e quatro agentes comunitários de saúde), que atuavam em unidades básicas de saúde (UBSs) localizadas na zona leste do município de São Paulo e que recebiam estudantes de Medicina do primeiro ano ao internato. As entrevistas foram gravadas e transcritas, lidas e relidas, sendo identificadas unidades de significado de acordo com os pressupostos da análise de conteúdo. Resultados: Os entrevistados destacaram as seguintes barreiras relacionados ao processo ensinoaprendizagem na APS: 1. excesso de pacientes agendados e escassez de tempo para discussão do caso com os estudantes; 2. inadequada estrutura física do serviço de saúde; 3. falta de preparação dos profissionais; $e$ 4. falta de articulação ensino-serviço-comunidade. Os aspectos reconhecidos como fatores facilitadores do processo ensino-aprendizagem foram: 1. qualificação do serviço de saúde e da equipe; 2. integração com a eSF e com equipe multiprofissional; e 3. preparação dos preceptores médicos. Conclusões: Os resultados têm implicações para os profissionais da APS, as instituições de ensino superior e os gestores da saúde da APS estudados. O aprimoramento das relações e articulações entre as instituições de ensino, os gestores dos serviços de saúde, os profissionais de saúde e a comunidade é condição necessária para efetivar o processo ensino-aprendizagem e garantir o desenvolvimento de competências para a qualidade do cuidado na APS. Assim, o preparo dos profissionais de saúde, a adequação do espaço físico da UBS, a reflexão sobre o agendamento e estratégias para garantir tempo para discussão do caso e espaço para feedback podem contribuir para mitigar as barreiras ao processo ensino-aprendizagem na APS. 


\section{KEYWORDS}

- Medical Education.

- Primary Health Care.

- Learning.

- Health Personnel.

- Community Health Workers.

- Qualitative Research.

\section{ABSTRACT}

Introduction: Although Primary Health Care (PHC) is essential for medical students' training, the perceptions of primary care workers about the teaching-learning process have been overlooked, particularly in municipalities where PHC management is performed by a private organization instead of the government, such as in the city of São Paulo. Objective: to analyze the perceptions of primary care workers about barriers and facilitators of medical students' teaching-learning process in PHC in the city of São Paulo. Method: we conducted a qualitative research. We performed in-depth interviews with 12 primary care workers from the family health teams (four physicians, four nurses and four community health workers), who worked in primary care clinics in the east region of the city and received medical students, from $1^{\text {st }}$-year to internship students. The interviews were recorded, transcribed and afterwards, they were repeatedly read. We identified thematic units following the content analysis principles. Results: the barriers to medical students' teaching-learning process in PHC were the following: (1) excessive number of scheduled patients and scarcity of time for discussion; (2) inadequate infrastructure of primary care clinics; (3) lack of training; and (4) ineffective integration among faculty, healthcare workers, managers and the assisted population. The facilitating factors of the teaching-learning process were: (1) high quality of healthcare services; (2) integration among primary care teams, interdisciplinary teams, and students; and (3) well-trained medical preceptors. Conclusions: our results have implications for PHC professionals, educational institutions, and managers. The improvement of the integration among educational institutions, health services managers, primary care workers, and the population is a condition to reach the effectiveness in the teaching-learning process, and to ensure the development of essential competencies for PHC assistance quality. Thus, the training of health professionals, improving the primary care clinic infrastructure, and creating strategies to ensure enough time for discussion and feedback could contribute to mitigate barriers to medical students' teaching-learning process in PHC.

Recebido em 3/12/19

Aceito em 12/3/20

\section{INTRODUÇ̃̃O}

O modelo de ensino-aprendizagem indicado pelas Diretrizes Curriculares Nacionais (DCN) para o curso de graduação em Medicina de 2014 propõe o aumento da carga horária dos estágios em atenção primária à saúde (APS) e postula que os conteúdos essenciais do curso de graduação em Medicina dialoguem com as principais necessidades de saúde identificadas na população, viabilizando-se uma inserção precoce dos estudantes em atividades práticas, bem como a utilização de diferentes cenários de ensino-aprendizagem ${ }^{1}$. A APS constitui um contexto de ensino-aprendizagem privilegiado para a compreensão das necessidades de saúde da população e para o aluno vivenciar um conjunto de ações vinculadas ao cuidado, desde o tratamento das doenças, a prevenção de agravos, promoção da saúde até a gestão em saúde, como trabalho em equipe, coordenação do cuidado, articulação das redes de atenção no Sistema Único de Saúde (SUS) ${ }^{2-4}$. Nesse sentido, as algumas contribuições da APS para a formação médica seriam: lidar com diferentes contextos e ciclos de vida da população assistida, suas complexidades clínica, social e cultural; ser um cenário de práticas em que ocorre a integração de diferentes campos do conhecimento e favorece a prática clínica integrada e interdisciplinar; possibilitar a compreensão da rede intersetorial de atenção à saúde; contribuir para o desenvolvimento da competência cultural e da comunicação em saúde; e lidar com problemas complexos de forma contínua e longitudinal ${ }^{4}$. Assim, os profissionais de saúde que atuam na APS são fundamentais para a formação de médicos na perspectiva da integralidade, equidade e humanização.

Em 1994, o Ministério da Saúde criou o Programa Saúde da Família para promover a reorganização da atenção básica no Brasil. As equipes de Saúde da Família (eSF) são constituídas por médicos, enfermeiros, técnicos de enfermagem e agentes comunitários de saúde (ACS), e atualmente são responsáveis pela cobertura de mais de 132 milhões de pessoas em todo o território nacional. Essas equipes recebem a maioria dos estudantes de Medicina tanto provenientes de instituições de ensino públicas como de instituições de ensino privadas, e esses profissionais assumem as atividades que esses discentes realizam na unidade básica de saúde (UBS), no território e nos domicílios da população adscrita ${ }^{2,5,6}$. Mesmo diante desse cenário, raros estudos investigaram as percepções dos profissionais das eSF sobre aspectos e/ou condições que podem afetar o processo ensino-aprendizagem dos estudantes de Medicina na APS. Uma pesquisa descreveu as percepções de médicos, enfermeiros, cirurgiõesdentistas, técnicos de enfermagem e ACS sobre a inserção dos estudantes de Medicina nas eSF no município de Montes Claros, em Minas Gerais. Um dos problemas descritos nos grupos focais foi o número elevado de estudantes por UBS, o que dificultou o planejamento da equipe e o vínculo dos profissionais com os estudantes ${ }^{5}$.

Uma condição que poderia ter implicações para o processo ensinoaprendizagem é o modelo de gestão dos serviços de atenção primária vigente no município, como a gestão direta das UBSs pela Secretaria Municipal de Saúde (SMS). Em Montes Claros, a contratação de profissionais para as eSF ocorre via concurso público, o que difere do modelo de gestão indireta, em que a SMS firma contratos com instituições privadas, como organizações sociais, para que estas gerenciem os serviços de APS, como ocorre no município de São Paulo7. Nesse caso, a contratação de profissionais para as eSF é realizada por essas instituições parceiras da SMS8. O modelo de gestão adotado pelo município pode afetar os cenários de ensino-aprendizagem na APS. Por exemplo, a alta rotatividade de médicos nas eSF do município de São Paulo, descrita na

REVISTA BRASILEIRA DE EDUCAÇÃO MÉDICA

2 44 (2) : e065; 2020 
pesquisa realizada por Campos e Malik8, pode ser uma barreira para as atividades desenvolvidas pelo estudantes de Medicina durante os estágios na APS9. Uma vez que a construção do cenário para o processo ensino-aprendizagem na APS depende intrinsecamente das articulações que se estabelecem entre as instituições de ensino superior, os gestores dos serviços de saúde, os profissionais de saúde que atuam nas UBSs e a população assistida ${ }^{2,5,10,11}$, torna-se fundamental investigar as percepções de profissionais das eSF sobre o processo ensino-aprendizagem em municípios nos quais o modelo de gestão da APS vigente é o indireto.

Desse modo, o objetivo desta pesquisa foi investigar as barreiras ou os facilitadores para o processo ensino-aprendizagem de estudantes de Medicina na APS, de acordo com as percepções de médicos, enfermeiros e ACS do município de São Paulo. Em relação aos estudos realizados previamente sobre o tema, a atual pesquisa acrescenta a investigação no cenário de ensino-aprendizagem na APS em um município cujo modelo de gestão dos serviços de APS é o modelo indireto, via instituições privadas denominadas organizações sociais. Além disso, esse é um dos raros estudos a investigar as percepções dos ACS sobre o processo ensinoaprendizagem dos estudantes de Medicina, apesar se serem profissionaischave para esse processo ${ }^{12}$. Assim, estudar as percepções dos ACS sobre o processo ensino-aprendizagem pode contribuir para aprimorar as relações ensino-serviço-comunidade e repercutir na qualidade do estágio. Busca-se aqui ampliar a compreensão dos aspectos que podem afetar o processo ensino-aprendizagem, as barreiras e os facilitadores, e, assim, contribuir para que as instituições de ensino superior, os gestores dos serviços de saúde e os profissionais de saúde possam elaborar ações para $o$ aprimoramento do processo ensino-aprendizagem.

\section{MATERIAL E MÉTODOS}

Conforme os objetivos do presente estudo, que busca compreender sentidos de fenômenos do mundo social ${ }^{13}$, optou-se por um desenho metodológico do tipo qualitativo. Segundo Minayo ${ }^{14}$, a metodologia qualitativa permite uma abordagem mais adequada para o estudo de objetos de pesquisa que se constituem por significados, motivos, aspirações, crenças, valores e atitudes, sobre os quais se estabelecem relações e processos complexos não redutíveis à perspectiva quantitativa.

A produção de dados ocorreu por meio de entrevistas com profissionais das equipes de saúde da família que atuam em UBSs da zona leste do município de São Paulo e recebem estudantes do primeiro ao $11^{\circ}$ semestre do curso de Medicina. Os sujeitos da pesquisa foram escolhidos de acordo com critérios qualitativos de seleção que os adjetivam como bons informantes-chave, ou seja, pessoas capazes de expressar bem suas opiniões e que são reconhecidas pelo seu protagonismo no ambiente em que atuam. Participaram da pesquisa 12 profissionais da saúde (quatro médicos, quatro enfermeiros e quatro ACS) das eSF da zona leste do município de São Paulo que atuam ativamente no processo ensinoaprendizagem, acompanhando os estudantes de Medicina durante os estágios. As UBSs em que esses profissionais trabalhavam eram geridas por uma instituição parceira da SMS, a Casa de Saúde Santa Marcelina.

As pesquisadoras foram uma aluna do internato médico e uma professora do módulo de Atenção Primária à Saúde e Medicina de Família e Comunidade, que não atuavam nas UBSs nas quais este estudo foi realizado e nem na organização social que geria esses serviços de saúde. Aos participantes foi apresentado o Termo de Consentimento Livre e
Esclarecido (TCLE), com garantias de privacidade e sigilo das entrevistas.

A pesquisa de campo foi realizada pela aluna, mediante treinamento e orientação para atuar como pesquisadora.

A técnica para produção de dados escolhida foi a entrevista em profundidade. Essa escolha se justifica por ser uma técnica que permite interação do pesquisador com o entrevistado, na qual o primeiro estimula a reflexão do segundo a propósito do que está sendo investigado. Nesse processo intersubjetivo, aprofunda-se o entendimento do pesquisador sobre a maneira como os entrevistados percebem e interpretam o objeto de estudo. De acordo com a técnica da entrevista em profundidade, o pesquisador realiza perguntas abertas, estimulando o entrevistado a discorrer sobre o tema proposto. $\mathrm{O}$ entrevistador tem a possibilidade de conduzir a discussão para o tema pesquisado e para os objetivos a serem alcançados, elucidando questões e esclarecendo dúvidas ${ }^{15}$. A técnica de entrevistas em profundidade também se mostrou operacionalmente mais viável neste estudo, tendo em vista os diferentes horários de trabalho dos profissionais, assim como a disponibilidade de tempo deles.

Elaboraram-se roteiros abertos de perguntas para as entrevistas (Anexo 1), considerando a tomada de perspectiva do tema por parte de usuários e profissionais da saúde. As entrevistas foram realizadas nas UBSs em horários pré-agendados, sendo gravadas e posteriormente transcritas e conferidas.

A análise dos dados se deu mediante leitura e releitura do material transcrito para identificação e agrupamento de unidades de significado e construção das categorias de análise, de acordo com a técnica de análise de conteúdo. Essa etapa da pesquisa foi realizada pelas três pesquisadoras. Consideraram-se os consensos e os dissensos de todas as intérpretes. As análises se deram pelo agrupamento temático do material empírico produzido a partir do material transcrito das entrevistas dos profissionais da saúde. Tais categorias estão dispostas no Quadro 1.

Este estudo é produto de pesquisa de Iniciação Científica vinculada ao Núcleo de Pesquisa em Atenção Primária e Ciência da Implementação (NUPAScience), do curso de Medicina da Faculdade Santa Marcelina. A pesquisa tem registros de aprovação pelo Comitê de Ética em Pesquisa da SMS de São Paulo (Parecer no 2.457.572) e pelo Comitê de Ética da Faculdade Santa Marcelina (Parecer no 2.423.305).

\section{RESULTADOS E DISCUSSÃO}

Foram entrevistados 12 profissionais de saúde (quatro médicos, quatro enfermeiros e quatro ACS) que atuavam nas eSF da zona leste do município de São Paulo e que recebiam e acompanhavam os estudantes

\section{Quadro 1}

Categorias temáticas a partir da leitura e releitura do material transcrito
Categorias temáticas

Barreiras para o processo ensino-aprendizagem

1. Agenda e tempo de consulta 2. Estrutura física do serviço de saúde 3. Falta de preparação dos profissionais 4. Falta de articulação ensino-serviço-comunidade

Facilitadores do processo 1. Qualificação do serviço de saúde e da equipe ensino-aprendizagem 2. Integração com a eSF e equipe multiprofissional 3. Preparação dos preceptores médicos
Fonte: Elaborado pelos autores

REVISTA BRASILEIRA DE EDUCAÇC̃̃ MÉDICA

3 44 (2) : e065; 2020 
de Medicina durante o estágio deles na APS. Todos esses profissionais eram contratados pela mesma organização social, a Casa de Saúde Santa Marcelina. Nove entrevistados foram mulheres. A idade desses profissionais variou de 25 a 44 anos, e a renda individual mensal, de $\mathrm{R} \$ 1.600,00$ a $18.000,00$

A partir da leitura e releitura do material transcrito, as seguintes categorias temáticas emergiram, tanto relacionadas às barreiras quanto às condições consideradas facilitadoras do processo ensino-aprendizagem.

\section{Barreiras para o processo ensino-aprendizagem na APS}

- Agenda e tempo restrito para realizar a consulta: Uma das barreiras sublinhadas pelos profissionais no aprendizado e formação dos acadêmicos diz respeito ao tempo para discussão dos casos e das condutas com os alunos, bem como para explanação de eventuais dúvidas e, sobretudo, da própria doença e de suas implicações. Nesse sentido, Kwa e Yong Rafidah ${ }^{16}$ postulam que o tempo escasso e as demandas conflitantes que podem advir dos estudantes e dos pacientes constituem desafios para o ensino na APS. No município de São Paulo, de acordo com as recomendações da SMS, os médicos possuem uma agenda fixa com o tempo de 15 minutos para realizar o atendimento do paciente, independentemente de a UBS receber alunos ou não.

[...] a maior dificuldade é a falta de tempo. A agenda do profissional não é uma agenda "protegida". A gente não tem tempo para discutir os casos, para ajudar, acompanhar todas as consultas. [...] as modificações [na agenda] não é uma coisa amparada pela prefeitura, pela Secretaria da Saúde, entendeu? Eles não dão autorização, a gente acaba fazendo uma adaptação, mas não consegue ter o tempo ideal para discutir os casos (Médico 3).

O tempo é muito corrido. Como as consultas programadas são 15 minutos só, é muito corrido. Tem acolhimento entre outras coisas (ACS2).

Eu acho que o tempo é uma dificuldade, porque a gente tem uma agenda muito fixa, né?, a cada 15 minutos, e muitas vezes é difícil já com esse tempo só a gente. E tem que explicar para um aluno ainda, gasta mais tempo com isso. Quando o médico é preceptor, deveria aumentar um pouco o tempo de consulta e diminuir o número de consultas que ele faz. O tempo é o limitador maior, com certeza (Médico 2).

- Estrutura física insuficiente: Os participantes deste estudo apontaram algumas limitações em relação à estrutura física nos serviços de APS no que concerne aos requisitos mínimos necessários para uma adequada ambiência para o processo ensino-aprendizagem. Tal problemática é descrita na literatura nacional. Em síntese, destacam-se a falta de estrutura adequada na UBS para acolher esses estudantes e o pouco tempo disponível para realização do atendimento médico.

"a estrutura física, acho que também é importante. A gente tem consultórios muito pequenos. Um número insuficiente de consultórios também" (Médico 3).

Para que a integração entre ensino e serviço seja bem-sucedida, são necessários alguns pré-requisitos tanto no convênio firmado entre a universidade e o serviço de saúde quanto na capacidade física da rede. A rede deve ser ampla, organizada e eficiente, situação muito distante da realidade que vivemos ${ }^{17}$. A falta de infraestrutura das UBSs dificulta o processo de ensino-aprendizagem ${ }^{9}$. Alguns autores destacaram a importância de prover espaço físico nos serviços de APS não apenas para assistir os pacientes, mas também para realizar discussões de caso mais aprofundadas e dar feedbacks aos estudantes com maior privacidade ${ }^{16}$.

- Falta de preparação dos profissionais que recebem os alunos: Alguns autores ressaltam a importância de haver pessoal suficiente, com capacitação e motivação adequadas para supervisão dos discentes, destacando a necessidade de preceptores com capacitação técnica, treinamento específico e que já detenham um perfil de atitudes que sirvam como exemplos para os futuros profissionais ${ }^{17}$.

Realizou-se uma pesquisa com gestores/coordenadores de cursos de Medicina em diversas regiões do Brasil, os quais relataram que o preparo inadequado de docentes é um fator que dificulta o desenvolvimento de competências dos estudantes para atuação na atenção primária ${ }^{9}$.

Entre os participantes do presente estudo, os ACS descreveram que não receberam nenhum tipo de preparação para o processo ensinoaprendizagem. De maneira geral, eles são apenas avisados quanto à chegada dos alunos e que farão atividades específicas com eles, como saída em visita domiciliar. Há qualificação e treinamento insuficientes dos profissionais responsáveis pela condução do processo ensinoaprendizagem, com destacou um dos participantes.

"Não. Treinamento a gente não recebe. O médico avisa antes[...]" (ACS 1).

- Falta de articulação ensino-serviço-comunidade: $\mathrm{O}$ vínculo é importante para que se estabeleçam laços interpessoais fundamentais para a qualidade do cuidado e para o profissional enxergar necessidades físicas, mentais e sociais, entre diversas outras características que contribuem para a melhora da qualidade do serviço prestado e da formação médica em $\mathrm{si}^{17}$. Apesar de os profissionais de saúde entrevistados descreverem a importância do vínculo de confiança que se estabelece em uma equipe e desta com os usuários da UBS, como menciona a enfermeira 4, a não comunicação com os usuários sobre a chegada e a presença dos alunos na UBS pode fragilizar o vínculo. Na prática, muitas vezes e repentinamente, o usuário é colocado na situação de ser atendido pelo estudante sem a contextualização prévia pelos profissionais das equipes, quebrando a expectativa do usuário de ter a consulta realizada pelo médico com o qual já estava vinculado. Isso gera estranhamento, desconforto e insatisfação do usuário (Enfermeira 1).

Aqui a gente tem o vínculo que é uma coisa muito forte da estratégia. A gente entra dentro da casa do paciente, a gente se insere na vida dele, dentro da família dele [...] (Enfermeira 4).

[...] tem paciente que, por exemplo, tem um vínculo bom com a doutora, aí chega e não vai passar com ela, passa com um aluno [...] eles [os pacientes] reclamam muito por isso [...] (Enfermeira 1).

Facilitadores do processo ensino-aprendizagem

- Qualificação do serviço de saúde e da equipe: Entende-se que a presença do estudante de Medicina na equipe melhora a qualidade do serviço, uma vez que incentiva os profissionais à prática reflexiva e os

REVISTA BRASILEIRA DE EDUCAÇÃO MÉDICA

4 44(2): e065; 2020 
estimula a pensar sobre seu raciocínio e suas decisões, fugindo de um estado de automatismo e pragmatismo na tomada de decisões. O modelo de atenção e ensino mais voltado para educação continuada privilegiaria a discussão dos casos com a equipe multiprofissional, alcançaria maior abrangência e justificativa de condutas, diminuiria erros e melhoraria o cuidado ${ }^{5}$. Médicos, enfermeiros e ACS que participaram deste estudo concordam com tais impactos, destacando que a presença do estudante qualifica o serviço e promove o desenvolvimento de características e habilidades anteriormente não notadas por parte da equipe.

[...] eles discutem as condutas conosco. Isso também dá um ganho de qualidade. Muitas vezes acabam vendo por uma ótica que nem o próprio preceptor percebeu [...] acaba dando uma riqueza maior de detalhes, uma riqueza maior de possibilidades. $E$ às vezes um ponto de vista, um olhar mais diferenciado [...] (Médico 1).

[...] toda vez que você vai fazer alguma coisa... Olha, tô fazendo isso por isso. Faz a gente voltar à teoria. Faz a gente meio que se reciclar. Você meio que tem que começar a justificar por que você tá fazendo aquilo [...] (Enfermeiro 1).

[...] quando tem aluno, o agente comunitário traz uma história mais embasada, com mais conteúdo, até para ele poder inserir, inteirar o estudante disso [...] (Enfermeira 3).

De acordo com os profissionais de saúde entrevistados, tal comportamento da equipe também se aplica na relação com a comunidade, destacando-se que, a partir da inserção de estudantes, a equipe passou a ser mais acolhedora e receptiva com os pacientes. Por conta desse cenário, a população se sente mais respaldada quando há a presença dos estudantes na UBS, pois associa isso a uma maior qualidade do serviço e dos profissionais e percebe que a instituição de ensino confiou no aprendizado de seus estudantes. Um estudo ${ }^{18}$ descreveu a percepção dos pacientes sobre ter alunos na UBS: os pacientes se sentiram mais bem acolhidos e receberam explanações melhores e mais minuciosas sobre sua doença e seu tratamento na presença dos estudantes. Essa melhora de qualidade na assistência foi atribuída à maior disponibilidade de tempo para o exame e às discussões sobre a doença entre o preceptor e os alunos durante $o$ atendimento.

[...] os pacientes gostam bastante porque você tem mais tempo para explicar sobre a patologia [...] eles se sentem mais assistidos, porque as consultas com os estudantes sempre demandam mais tempo [...] eles se sentem valorizados, acolhidos ...] (Médico 4).

Os ACS também relataram que a presença dos estudantes nas visitas domiciliares é positiva, pois traz credibilidade junto à população que se sente mais segura quando o ACS é acompanhado pelo aluno, contribuindo para a melhora do vínculo serviço-comunidade ${ }^{19}$ :

Traz bastante benefício. Traz credibilidade junto à população, $e$ nos ajuda a fazer busca ativa [...] é positivo [...] (ACS 1).

Além disso, verifica-se uma construção de conhecimento e significado na presença do estudante no cotidiano do ACS, o que contribui para a educação em saúde desses profissionais, já que se trata de um grupo com muitas indefinições relacionadas às habilidades, às competências e aos saberes, e, em grande parte, marginalizado quanto à qualificação e ao treinamento em saúde ${ }^{19}$ :

Tem horas que eles trazem dúvidas com a visão deles como estudante e acaba solucionando para a gente também [...] (ACS 3).

- Integração com a eSF e equipe multiprofissional: Alguns autores destacam que o diálogo entre o trabalho e a educação se dá em espaços em que o eixo ensino-serviço-comunidade estabelece confluência de saberes e modos de ver o mundo ${ }^{20}$. Um estudo avaliou a percepção dos alunos de Medicina sobre o estágio na APS, e os participantes referiram a importância da APS no processo de formação médica ${ }^{21}$. A integração do estudante de Medicina precocemente inserido na comunidade contribui para a formação de um profissional mais completo, ciente e partícipe da realidade social encontrada nas periferias e capaz de integrar uma equipe multiprofissional.

[...] depois que os alunos começaram a frequentar a unidade, a equipe mudou a postura também. A equipe entendeu melhor qual é a função desse aluno dentro da equipe [...] e os alunos se integram muito bem (Médico 3).

Para os profissionais entrevistados, a integração dos alunos e da equipe ocorre de forma efetiva, com os profissionais entendendo a função do aluno dentro da equipe. Observam ainda uma transformação do profissional médico que passa a ter um "olhar diferenciado" do seu poder de efetividade e de qualidade de atendimento.

Do ponto de vista de formação do profissional médico na eSF, buscase o desenvolvimento interdisciplinar que envolva todos os saberes de maneira igualmente importante, com melhoria permanente da qualidade do cuidado à saúde e da humanização do atendimento. A interdisciplinaridade é vivenciada pelos alunos nos grupos de promoção da saúde, em que o estudante é inserido no universo da comunidade, na interação com os ACS em visitas domiciliares, nas consultas médicas e de enfermagem.

\section{[...] é bastante importante ensinar o relacionamento em uma equipe multiprofissional, não que o aluno seja formado para trabalhar no ambiente médico-centrado, mas sim sempre em uma equipe multiprofissional. Que ele aprenda a respeitar o papel dos outros profissionais e que possa contribuir com eles e aceitar ajuda deles quando é necessário (Médico 1).}

A reformulação do ensino médico preconiza maior capacidade de integração com a comunidade e aquisição de competências socioculturais. O contexto de imersão do aluno na comunidade, especialmente por meio das visitas domiciliares com o ACS, mostra um mundo muitas vezes desconhecido e distante da realidade do acadêmico, transformando seu olhar para uma visão mais ampla e completa da realidade médica e social.

[...] eles criam vínculo também. Começam a entender um problema [...] o problema que a comunidade, que a sociedade tem. Que muitos não moram, não residem nesses lugares [...] eles acabam pegando um pouco desse problema social, querendo ajudar [...] (ACS 2).

- Preparação dos preceptores: Quando os profissionais entrevistados foram questionados sobre treinamentos ou qualificações específicas, apenas os profissionais médicos afirmaram ter feito alguma

$5 \mid$\begin{tabular}{l|l} 
REVISTA BRASILEIRA DE EDUCAÇÃO MÉDICA \\
\hline 44 (2) : e065; 2020
\end{tabular} 
preparação antes da chegada dos estudantes. Estes também revelaram que participam de reuniões mensais, com cursos de aprimoramento e educação continuada para exercer a preceptoria.

[...] a gente foi informado e a gente fez toda uma preparação antes de recebê-los na época (Médico 3).

[...] a gente tem reuniões mensais com supervisores, com os professores diretos [...] estamos sempre em reunião, sempre em curso de aprimoramento (Médico 4).

Limitações do estudo

O presente estudo possui limitações, como o fato de os participantes serem contratados pela mesma organização social, a Casa de Saúde Santa Marcelina, que tem tradição em ensino e pesquisa nos serviços de APS da zona leste do município de São Paulo. Desse modo, não foram analisadas percepções de profissionais que atuam em outras instituições parceiras da SMS e em outras regiões do município. Outra limitação é a não realização de triangulação por meio da análise das percepções de estudantes, docentes da faculdade e gestores.

\section{CONSIDERAÇÕES FINAIS}

A inserção do estudante de Medicina na APS propicia a aquisição e o desenvolvimento de habilidades e qualidades essenciais na formação de um profissional médico para prestar cuidado efetivo, integral e humanístico, pois os profissionais de saúde que atuam na APS são elementos-chave para o processo ensino-aprendizagem. Apesar disso, raros foram os estudos que analisaram as percepções desses atores sobre esse processo. Em particular, encontramos apenas um estudo que incluiu os ACS para analisar percepções de profissionais sobre a inserção dos alunos na APS. Além disso, os municípios possuem autonomia para decidir qual modelo de gestão de serviços de APS devem adotar, se direto ou indireto, o que pode ter repercussões nos cenários de práticas. Esta pesquisa estudou as percepções de usuários, agentes comunitários, médicos e enfermeiros que atuam na atenção primária do município de São Paulo, que tem um modelo de gestão indireta da APS, sobre barreiras e facilitadores do processo ensino-aprendizagem de estudantes de Medicina.

Diante das barreiras para o processo ensino-aprendizagem identificadas nesta pesquisa, as estratégias para superá-las incluem, primeiramente, promover a revisão da estrutura física adequada, a gestão dos espaços para assistência, como otimização do mapa de salas das UBSs, para a discussão de casos e para realização dos feedbacks periódicos para os estudantes, bem promover a discussão e a elaboração de uma agenda protegida e direcionada para o ensino, reservando o tempo necessário para discussão do caso, evitando a sobrecarga dos profissionais das eSF envolvidos no processo ensino-aprendizagem e as queixas dos pacientes pelo tempo de espera. $\mathrm{O}$ aprimoramento das relações e articulações entre as instituições de ensino e os gestores dos serviços de saúde é condição necessária para efetivar o processo ensino-aprendizagem e garantir o desenvolvimento de competências para a qualidade do cuidado na APS. A presença de profissionais, não só médicos, qualificados e capazes de contribuir efetivamente para a educação médica é necessidade frequentemente negligenciada, que acaba levando o ensino de volta ao modelo médico-centrado. Outro ponto fundamental é a integração com ensino-serviço-comunidade, melhorando a compreensão dos usuários sobre a importância de ter estudantes em um serviço de saúde e seus significados, além de prover espaços de conversa para que os usuários exponham suas dúvidas e reflexões sobre a presença do aluno e o processo ensino-aprendizagem no qual o aluno, o profissional de saúde e o usuário estão inseridos. Desse modo, os resultados trazem repercussões para docentes, profissionais de saúde que recebem alunos, gestores dos serviços de saúde e gestores da educação médica, e descrevem pontos-chave para a melhora da integração ensino-serviço-comunidade em uma região do município de São Paulo.

\section{AGRADECIMENTOS}

Agradecemos à equipe de Atenção Primária à Saúde da Casa de Saúde Santa Marcelina e à Secretaria Municipal de Saúde do município de São Paulo, em especial à Coordenadoria Regional de Saúde Leste. Este estudo é produto de pesquisa de Iniciação Científica vinculada ao Núcleo de Pesquisa em Atenção Primária e Ciência da Implementação (NUPAScience), do curso de Medicina da Faculdade Santa Marcelina.

\section{REFERÊNCIAS}

1. Brasil. Conselho Nacional de Educação. Câmara de Educação Superior. Resolução CNE/CES no 3, de 20 de junho de 2014. Institui Diretrizes Curriculares Nacionais do Curso de Graduação em Medicina e dá outras providências. Diário Oficial [da] República Federativa do Brasil, Brasília, DF; 23 jun 2014; Seção 1, p. 8-11 [acesso em 16 nov 2018]. Disponível em: http://portal.mec.gov.br/index. php?option=com_docman \&view $=$ download $\&$ alias $=15874$-rces00314\&Itemid=30192.

2. Vieira JE, Elias PEM, Benseñor IJM, Grisi SJE. Instalação da disciplina de Atenção Básica em Saúde na Faculdade de Medicina da Universidade de São Paulo (2003-2006). Rev. bras. educ. med. 2007;31(3):236-44 [acesso em 10 jul 2018]. Disponível em: http://www.scielo.br/scielo. php?script=sci_arttext\&pid=S0100-55022007000300006\&lng=en\&nr $\mathrm{m}=\mathrm{iso} \& \mathrm{t} \operatorname{lng}=\mathrm{pt}$.

3. Inez $\mathrm{M}$, Anderson P, Demarzo M, Rodrigues RD. A medicina de família e comunidade, a atenção primária à saúde e o ensino de graduação. Rev. bras. med. fam. comunidade 2007;3(11):1-32.

4. Demarzo MMP, Almeida RCC de, Marins JJN, Trindade TG da, Anderson MIP, Stein AT, et al. Diretrizes para o ensino na atenção primária à saúde na graduação em medicina. Rev. bras. educ. med 2012;36(1):143-8 [acesso em 14 set 2018] Disponível em: http://www.scielo.br/scielo.php?script=sci_arttext\&pid=S0100$55022012000100020 \& \operatorname{lng}=$ en\&nrm $=$ iso\&tlng $=$ pt.

5. Caldeira ÉS, Leite MT de S, Rodrigues-Neto JF. Estudantes de Medicina nos serviços de atenção primária: percepção dos profissionais. Rev. bras. educ. med. 2011;35(4):477-85 [acesso em 12 fev 2019]. Disponível em: http://www.scielo.br/scielo.php?script=sci_ arttext\&pid=S0100-55022011000400006.

6. Brandão ERM, Rocha SV, Silva SS da. Práticas de integração ensinoserviço-comunidade : reorientando a formação médica. Rev. bras. educ. med. 2013;37(4):573-7.

7. Coelho VSP, Greve J. As organizações sociais de saúde e o desempenho do SUS: um estudo sobre a atenção básica em São 
Paulo. Dados 2016; 59(3):867-901 [acesso em 6 out 2019]. Disponível em: http://www.scielo.br/scielo.php?script=sci_arttext\&pid=S001152582016000300867\&lng=pt\&tlng=pt.

8. Campos CV de A, Malik AM. Satisfação no trabalho e rotatividade dos médicos do Programa de Saúde da Família. Rev Adminstração Pública 2008;42(2):374-68.

9. Vieira S de P, Pierantoni CR, Magnago C, Ney MS, Miranda RG de. A graduação em Medicina no Brasil ante os desafios da formação para a atenção primária à saúde. Saúde debate 2018;42:189-207.

10. Silva ATC da, Medeiros Junior ME de, Fontão P de N, Saletti Filho HC, Vital Junior PF, Bourget MMM, et al. Medicina de Família do primeiro ao sexto ano da graduação médica: considerações sobre uma proposta educacional de integração curricular escola-serviço. Rev. bras. educ. med. 2017;41(2):336-45 [acesso em 3 jan 2018]. Disponível em: http://www.scielo.br/scielo.php?script=sci_arttext\&pid=S010055022017000200336\&lng=pt\&tlng=pt.

11. Cassel C, Wilkes M. Location, location, location: where we teach primary care makes all the difference. J. gen. intern. med. 2017;32:4115 [acesso em 15 abr 2019]. Disponível em: http://link.springer. com/10.1007/s11606-016-3966-x.

12. Pereira JG, Regina W, Martines V, Chueiri PS. Integração academia, serviço e comunidade: um relato de experiência do curso de graduação em Medicina na atenção básica no município de São Paulo. Mundo saúde 2009;33(1):99-107.

13. Neves J. Pesquisa qualitativa: características, usos e possibilidades. Cad Pesq em Adm São Paulo 1996;1(3):1-5.

14. Minayo MC de S. O desafio do conhecimento: pesquisa qualitativa em saúde. São Paulo: Hucitec; 1993.

15. Boni V, Quaresma S. Aprendendo a entrevistar: como fazer entrevistas em Ciências Sociais. Em Tese 2005;2(3):68-80.

16. Kwa S, Yong Rafidah A. Notes for the primary care teacher: teaching in the family practice clinics. Malays Fam Physician 2008;3(2):1013 [acesso em $1^{\circ}$ out 2019]. Disponível em: http://www.ncbi.nlm.nih. gov/pubmed/25606127.

17. Troncon LE de A. Ensino clínico na comunidade. Med Ribeirão Preto 1999;32:335-44.

18. Berwanger J, Denti De Geroni G, Bonamigo EL. Estudantes de Medicina na percepção dos pacientes. Rev. bioét. 2015;23(3):55262 [acesso em 20 nov 2018]. Disponível em: http://dx.doi. org/10.1590/1983-80422015233092.

19. Pedraza DF, Santos I. Perfil e atuação do agente comunitário de saúde no contexto da Estratégia Saúde da Família em dois municípios da Paraíba. Interações 2017;18(3):97-105 [acesso em 15 mar 2018]. Disponível em: http://www.scielo.br/pdf/inter/v18n3/1518-7012inter-18-03-0097.pdf

20. Albuquerque VS, Gomes AP, Rezende CHA de, Sampaio MX, Dias OV, Lugarinho RM. A integração ensino-serviço no contexto dos processos de mudança na formação superior dos profissionais da saúde. Rev. bras. educ. med. 2008;32(3):356-62.
21. Massote AW, Belisário SA, Gontijo ED. Atenção primária como cenário de prática na percepção de estudantes de Medicina. Rev. bras. educ. med. 2011;35(4):445-53.

\section{CONTRIBUIÇÃO DOS AUTORES}

Moniquelly Barbosa da Silva realizou a revisão bibliográfica, a coleta dos dados, a transcrição do material das entrevistas, a análise dos dados e a interpretação e escrita do manuscrito. Izabel Rios realizou a análise dos dados e interpretação dos achados, e a escrita e revisão do manuscrito. Pedro Félix Vital Júnior realizou a análise dos dados, a interpretação dos resultados e a escrita do manuscrito. Andréa Tenório Correia da Silva elaborou e supervisionou a pesquisa, e realizou a análise do material transcrito, a interpretação dos resultados e a escrita e revisão do manuscrito.

\section{CONFLITO DE INTERESSES}

Os autores declaram não haver conflito de interesses neste estudo.

\section{ENDEREÇO PARA CORRESPONDÊNCIA}

Andréa Tenório Correi da Silva. I. Faculdade Santa Marcelina, Núcleo de Pesquisa em Atenção Primária e Ciência da Implementação (NUPAScience). Rua Cachoeira de Itupanema, 40 - Vila Carmosina, São Paulo - SP, 08270-140. II. Departamento de Medicina Preventiva, Faculdade de Medicina da Universidade de São Paulo. Av. Dr. Arnaldo, 455, Cerqueira César, São Paulo - SP, CEP 01246903. Departamento de Medicina Preventiva, Sala 2221.

E-mail: andreatenorio@usp.br

Anexo 1

Roteiros de entrevista em profundidade

\section{Roteiro da entrevista com os profissionais de saúde}

(1) Na sua opinião, como é ter um estudante de Medicina na sua equipe de saúde da família?

(2) Você recebeu alguma orientação para receber os estudantes de Medicina na sua equipe?

(3) Você acha que a presença dos estudantes de Medicina na equipe trouxe alguma mudança? Por quê?

(4) $\mathrm{Na}$ sua opinião, quais são as dificuldades para o ensino dos estudantes de Medicina na unidade básica de saúde?

(5) Na sua opinião, o que poderia ser melhorado para favorecer o ensino dos estudantes de Medicina?

(6) O que você acha que a unidade básica de saúde deve ensinar para o estudante de Medicina para ele ser um bom médico?

(7) O que você acha que poderia ser modificado para que o aluno aprenda melhor? 